\title{
Incarcerated sigmoid colon with gangrenous appendices epiploicae: A rare case report
}

\author{
Wadhawan G. ${ }^{1}$, Vyas K.C. ${ }^{2}$ \\ ${ }^{1}$ Dr. Gaurav Wadhawan, Assistant Professor, ${ }^{2}$ Dr. K. C. Vyas, Professor and Head, Department of Surgery, Pacific \\ Medical College and Hospital, Udaipur, Rajasthan, India. \\ Corresponding Author: Dr. Gaurav Wadhawan, Assistant Professor, Department of Surgery, Pacific Medical College \\ and Hospital, Udaipur, Rajasthan, India. Email: gauravwadhawan9@gmail.com
}

\begin{abstract}
Incarcerated inguinal hernia is a common diagnosis in patients presenting with a painful and not reducible groin mass. Sliding hernias are supposed to be more anatomically challenging for a surgeon than an uncomplicated inguinal hernias. The anatomical and physiological concept of sliding inguinal hernia is frequently misunderstood by surgeons of all levels of experience. Except in sliding hernia, the sigmoid colon is uncommonly found in an inguinal hernia.
\end{abstract}

Keywords: Incarcerated Inguinal Hernia left side, Sigmoid colon, Appendices epiploicae

\section{Introduction}

A sliding inguinal hernia is a protrusion of retroperitoneal organ through an abdominal wall defect and these are usually direct inguinal hernias containing various abdominal viscera. It is estimated that $75 \%$ of all hernia occur in the inguinal region [1]. Frequency of sliding hernia is estimated at $3-8 \%$ of all elective operations of inguinal hernias. Inguinal hernias have a nature to surprise with its unexpected contents. Appendix epiploicae in the hernia sac is a rare entity and that too if hypertrophied and presenting as irreducible hernia is still more uncommon. The content of the hernia sac may vary and nearly all the abdominal organs have been found within hernia sac [2]. Epiploic appendices were first described by Vesalius in 1543 as anatomic entities along the antimesenteric teniaecoli [3]. Hypertrophied and strangulated epiploic appendix in the sac of inguinal hernia is very rare and not many cases have been reported in the literature. The medline search shows case reported from Turkey in 1989 and Russia in 2005 [4,5]. We present an unusual case of incarcerated left inguinal hernia containing sigmoid colon with abnormal hypertrophied growth of appendices epiploicae engulfing another gangrenous appendices epiploicae in it.

\section{Case Report}

A 58 year old male was admitted with complain of painful swelling left groin since last 10 days, he had the history of swelling since last one year which was reducible. No previous history of any surgery.

Per abdominal examination was soft, non -tender, bowel sounds present, there was a tender non reducible swelling in the left inguinoscrotal region, cough impulse negative with feeble gurgling sound on auscultation, could not reach above the swelling in scrotum, left testis was palpable separately non tender, transillumination was negative. X-ray abdomen was normal; CT scan showed that there is herniation of sigmoid colon with abdominal fat through a defect into left inguinal region. The abdominal fat is seen reaching up to the left scrotal sac along with small loculated collection.

The patient was investigated and taken for surgery, intraoperatively it was found that it was a direct incarcerated sliding inguinal hernia with sigmoid colon and its appendices epiploicae one of which was hypertrophied into a mass engulfing other appendices (fig1) which was twisted around it and was gangrenous with seropurulent fluid around its attachment in the scrotal sac.

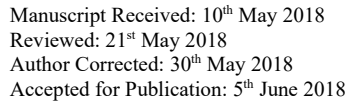




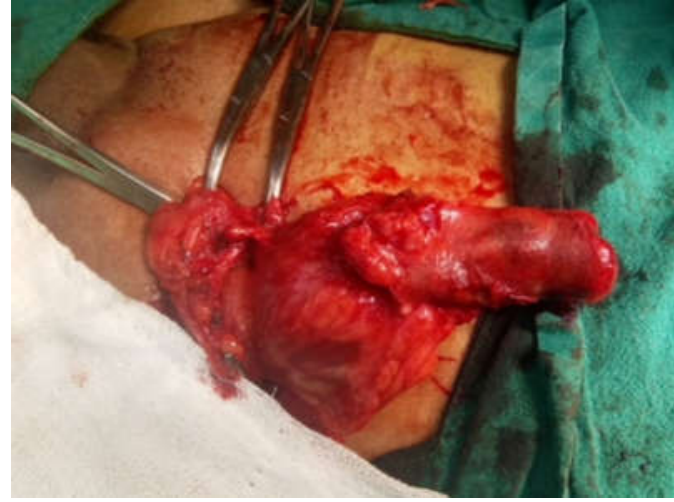

Fig-1: Hypertrophied appendices epiploicae

\section{Case Report}

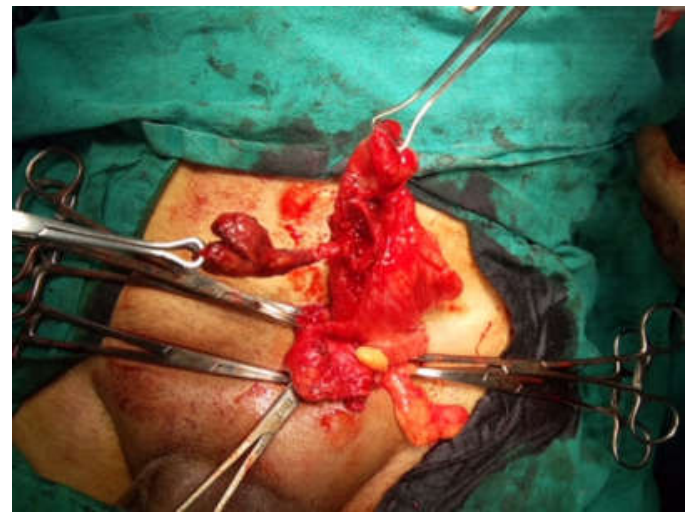

Fig-2: Gangrenous appendices epiploicae

The contents of the sac were dissected, the gangrenous appendices epiploicae (fig2) was excised and sent for histopathology, contents were reduced and hernioplasty done with prolene mesh. The post- operative period was uneventful and patient was discharged in a week in satisfactory condition. He is being followed up in the outpatient department.

\section{Discussion}

Although the first description of sliding inguinal hernia is often attributed to Galen, the first precise, modern description of sliding hernia accentuating "specificity" of this condition was made by Antonia Scarpa (17521832), Italian Anatomist and surgeon professor at the Universityof Pavia in northern Italy. In his work "Sull'ernie" (on hernias) he described in details the sliding variant of inguinal hernia [6]. In adults virtually all cases of sliding hernias are seen in males [7]. Incarceration of inguinal hernias occur in $10 \%$ of cases which in turn can lead to obstruction, strangulation and infarction [8].Among these complications, strangulation is the most serious with potentially lethal sequelae [9].

The content of inguinal hernias varies widely but in our case the contents of the hernia sac was the incarcerated loop of sigmoid colon probably due to redundancy. To our knowledge we were seeing that unusual presentation of Appendices epiploicae for the first time; they were hypertrophied into a tubular mass engulfing and encircling other gangrenous appendices.

Appendices epiploicae are fat containing peritoneal out pouching's arising from the serosal surface of the colon, found at any point between caecum and recto sigmoid colon. The length may vary from 0.5 and $5 \mathrm{~cm}$ [10]. These may have bacteriostatic properties, may act as a cushion to blood vessels and may perform absorptive action of the large bowel.

Appendix epiploicae as a content of the inguinal hernia sac is a rare phenomenon and very few cases have been reportedby now $[4,5]$. Further hypertrophied and inflamed or gangrenous epiploicae as content of inguinal hernia is also more rarely seen [11]. Earlier cases on relation of epiploicae appendices and hernia have been reported from USA as early as 1920 [12]. Mayank Jain has reported a case of epiploic appendagitis as an inguinal hernia content which was treated laparoscopically [11]. Huseyin Ozkurt has described CT scan as diagnostic investigation modality in case of epiploic appendagitis, one of this case had incarcerated inguinal hernia with inflamed and oedematous appendix epiploicae of the sigmoid colon in the hernia sac [13]. Traditionally sliding hernia was considered difficult to operateon [14].

Our patient had hypertrophied and gangrenous appendix epiploicae of the sigmoid colon incarcerated in the inguinal hernia sac which was removed and the inguinal hernia repaired. The patient recovered well after being discharged from the hospital and was followed regularly post operatively with uneventful progress.

\section{Conclusion}

Inguinal hernia is a common clinical condition which may be having varied contents in its hernial sac and the list of contents is constantly increasing. We came across an altogether different presentation of Appendices epiploicae which we thought to share. Timely presentation of the patient to the doctor and timely surgery helps in decreasing the mortality and morbidity of any acute cases which should not be neglected.

Conflict of interest: None declared.

Funding: Nil, Permission from IRB: Yes 


\section{Case Report}

\section{References}

1. Malangoni MA, Rosen MJ. Hernias In: Townsend CM, Beauchamp RD, Evers BM, Mattox KL (Eds.). Sabiston textbook of surgery, Saunders Elsevier, Philadelphia 2008; 18:1155-1179.

2. Girotto JA, Shaikh AY, Freeswick PD, et al. Diverticulitis presenting as a strangulated inguinal hernia. DOI:10. 1159/000052011

3. De Vesalius A. Humanis corporis fabricia libri septem. In: Basileae, ed. Andreaevesaliibruxellensis, schloaemedicorumpatauiniae professoris de humani corporis fabricialibriiseptem. Basel, Switzerland: Ex officinal Joannis Oporini; 1543-4.

4. Abdulzhavadov IM. Volvulus of the epiploic appendices of the sigmoid in strangulated inguinal hernia. Klin Med (Mosk). 1989 Jan;67(1):126-7.

5. Kulacoglu H, Tumer H, Aktimur R, Kusdemir A. Epiploic appendicitis in inguinal hernia sac presenting an inguinal mass. DOI: 10. 1007/s10029-004-03 06-6

6. Scarpa A. Sull'ernie: memorieanatomo-chirurgiche. Milan: StamperiaReale, 1809,1820.

7. RYAN EA. An analysis of 313 consecutive cases of indirect sliding inguinal hernias. SurgGynecol Obstet. 1956 Jan;102(1):45-58.

8. Surg Gyn Obst1956; 102:45-58.
9. Mc Fadyen BV Jr, Mathis CR. Inguinal herniorrhaphy: complications and recurrences. Semin Laparosc Surg 1994; 1:128-40.

Mac Fadyen BV Jr, MathisCR. Inguinal Herniorrhaphy: Complications and Recurrences. DOI: 10.1053/ SLA S00100128

10. Gallegos NC, Dawson J, Jarvis M, Hobsley M. Risk of strangulation in groin hernias. Br J Surg. 1991 Oct; 78 (10):1171-3

11. Bastidas JG, Danzy LE, Blackwell L, et al. Epiploic appendagitis in a 24-year-old woman. DOI:10.1016/j. ajem.2008.01.033

12. Jain M, Khanna S, Sen B, Tantia O. J Minim Access Surg. 2008 Jul;4(3):85-7.

13. Rosenstock J, Hansen L, Zee P, Li Y, et al. Dual add-on therapy in type 2 diabetes poorly controlled with metformin monotherapy: a randomized double-blind trial of saxagliptin plus dapagliflozin addition versus single addition of saxagliptin or dapagliflozin to metformin. DOI:10.2337/dc14-1142

14. Ozkurt H, Karatağ O, Karaarslan E, Başak M. Clinical and CT findings of epiploic appendagitis within an inguinal hernia. Diagn IntervRadiol. 2007 Mar;13(1): 23-5.

15. Bendavid R. Sliding hernias. DOI:10.1007/s10029002-0065-1

\section{How to cite this article?}

Wadhawan G, Vyas K.C. Incarcerated sigmoid colon with gangrenous appendices epiploicae: A rare case report. Surgical Update: Int J surg Orthopedics. 2018;4(2):87-89. doi:10.17511/ijoso.2018.i02.05. 